\title{
Correction: Immunogenicity of mammary tumor cells can be induced by shikonin via direct binding-interference with hnRNPA1
}

\author{
Shu-Yi Yin ${ }^{1}$, Thomas Efferth ${ }^{2}$, Feng-Yin Jiann ${ }^{1}$, Yung-Hsiang Chen ${ }^{1}$, Chia-I Liu ${ }^{3}$, \\ Andrew H.J. Wang ${ }^{4}$, Yet-Ran Chen ${ }^{1}$, Pei-Wen Hsiao ${ }^{1}$ and Ning-Sun Yang ${ }^{1}$ \\ ${ }^{1}$ Agricultural Biotechnology Research Center, Academia Sinica, Taipei, Taiwan, ROC \\ 2 Institute of Pharmacy and Biochemistry, University of Mainz, Germany \\ ${ }^{3}$ School of Medical Laboratory Science and Biotechnology, Taipei Medical University, Taipei, Taiwan, ROC \\ ${ }^{4}$ Institute of Biological Chemistry, Academia Sinica, Taipei, Taiwan, ROC \\ Published: August 14, 2018
}

Copyright: Yin et al. This is an open-access article distributed under the terms of the Creative Commons Attribution License 3.0 (CC BY 3.0), which permits unrestricted use, distribution, and reproduction in any medium, provided the original author and source are credited.

This article has been corrected: An institutional investigation was conducted at the request of the Oncotarget editorial staff by the Academia Sinica Ethics Committee (Taipei, Taiwan). The Committee concluded the following: "The partial duplication of Figure $4 \mathrm{a}$ and $4 \mathrm{~b}$ is judged to be a mistake during data processing, rather than a research misconduct.” The correct Figure 4B is shown below; 4A appears correctly in the original article. The authors declare that these corrections do not change the results or conclusions of this paper.

b MDA-MB231 MDA-MB231-hnRNP A1
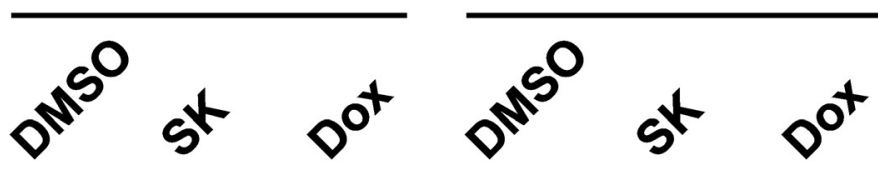

HSP70

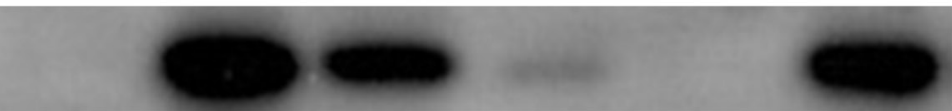

$470 \mathrm{kDa}$

HMGB1
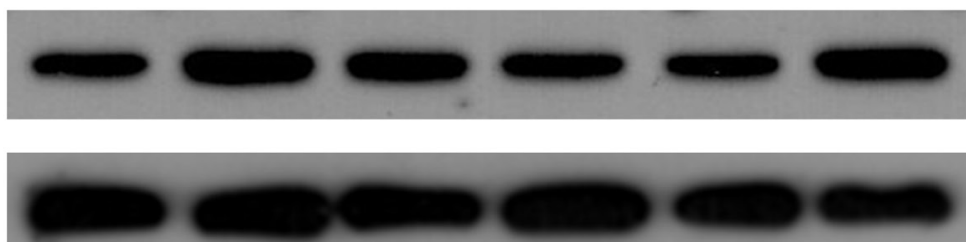

\section{$29 \mathrm{kDa}$}

\section{Actin}
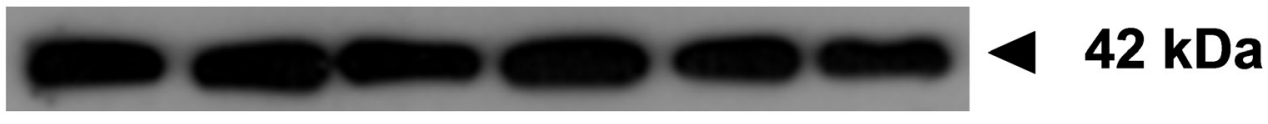

Figure 4: hnRNPA1 is a critical mediator of the SK-induced ICD activity in mammary tumor cells. a. and b. Western blot analyses of expression of HSP70 and HMGB1 in human (MDA-MB-231) and mouse (4T1) tumor cells. Some 4T1, 4T1-hnRNPA1, MDAMB-231 and MB-231-hnRNPA1 cells were treated with SK or Dox at $5 \mu \mathrm{g} / \mathrm{ml}$ for $24 \mathrm{~h}$. $\beta$-actin was used as a loading control.

Original article: Oncotarget. 2016; 7:43629-43653. https://doi.org/10.18632/oncotarget.9660 